\title{
Finite Metabelian Group Algebras
}

\author{
Shalini Gupta \\ Department of Mathematics, Punjabi University, Patiala, India \\ email:gupta_math@yahoo.com
}

Keywords: semisimple group algebra, primitive central idempotents, Wedderburn decomposition, metabelian groups.

\begin{abstract}
Given a finite metabelian group G, whose central quotient is abelian (not cyclic) group of order $p^{2}, p$ odd prime, the objective of this paper is to obtain a complete algebraic structure of semisimple group algebra $\mathbb{F}_{q}[G]$ in terms of primitive central idempotents, Wedderburn decomposition and the automorphism group.
\end{abstract}

\section{Introduction}

Let $F$ be a field and $G$ be a finite group such that the group algebra $F[G]$ is semisimple. A fundamental problem in the theory of group algebras is to understand the complete algebraic structure of semisimple group algebra $F[G]$. In the recent years, a lot of work has been done to solve this problem $[1,2,5,7,8,9]$. Bakshi et.al [3] have solved this problem for semisimple finite metabelian group algebras $\mathbb{F}_{q}[G]$, where $\mathbb{F}_{q}$ is a finite of order $q$ and $G$ is a finite metabelian group. They further illustrated their algorithm by explicitly finding a complete set of primitive central idempotents, Wedderburn decomposition and the automorphism group of semisimple group algebra of certain groups whose central quotient is Klein's four-group. In the present paper, a complete algebraic structure of semisimple group algebra $\mathbb{F}_{q}[G]$ for some finite groups $\mathrm{G}$, whose central quotient, $G / Z(G)$, is the direct product of two cyclic groups of order $p, p$ odd prime, is obtained. It is known [6] that finitely generated groups $G$, whose central quotient is isomorphic to $\mathbb{Z}_{p} \times \mathbb{Z}_{p}$ break into nine classes. The complete algebraic structure of $\mathbb{F}_{q}[G]$, for group $G$ in the two of the nine classes, is obtained in the present paper.

\section{Notation}

Let $\mathrm{G}$ be a finite group of order coprime to $q$ and $\operatorname{Irr}(G)$ denotes the set of all irreducible characters of $G$ over $\overline{\mathbb{F}}_{q}$, the algebraic closure of $\mathbb{F}_{q}$. Let $H \triangleleft K \leq G$ such that $K / H$ is cyclic of order $n$ and $T=N_{G}(H) \cap N_{G}(K)$, where $N_{G}(H)$ denotes the normalizer of $H$ in $G$. Let $\mathcal{C}(K / H)$ denotes the set $q$-cyclotomic sets of $\operatorname{Irr}(K / H)$ containing the generators of $\operatorname{Irr}(K / H)$. Suppose that $T$ act on $\mathcal{C}(K / H)$ by conjugation, then it is easy to see that stabilizer of any $C \in \mathcal{C}(K / H)$ remains the same. Let $E_{G}(K / H)$ denotes the stabilizer of any $C \in \mathcal{C}(K / H)$ and let $\mathfrak{R}(K / H)$ denotes the set of distinct orbits of $\mathcal{C}(K / H)$ under the action of $T$ onC $(K / H)$. Observe that

$$
|\mathfrak{R}(K / H)|=\frac{\phi(n)\left|E_{G}(K / H)\right|}{|T| \operatorname{ord}_{n}(q)},
$$

where $\operatorname{ord}_{n}(q)$ denotes the order of $q$ modulo $n$. 
For $C \in \mathcal{C}(K / H), \chi \in C$ and $\zeta_{n}$ a primitive nth root of unity in $\overline{\mathbb{F}}_{q}$, set

$$
\varepsilon_{C}(K / H)=|K|^{-1} \sum_{g \in K}\left(t r_{F_{q}\left(\zeta_{n}\right) / F_{q}}(\chi(\bar{g}))\right) g^{-1},
$$

and $e_{C}(G, K, H)$ as the sum of distinct $G$-conjugates of $\varepsilon_{C}(K / H)$.

\section{Metabelian group algebras}

The notation used in [4] will be followed: For a normal subgroup $N$ of $G$, let $A_{N} / N$ be an abelian normal subgroup of $G / N$ of maximal order. Let $D_{N}$ be the set of subgroups $D / N$ of $A_{N} / N$ such that $A_{N} / D$ is cyclic and $T_{G / N}$ be the set of representatives of $D_{N}$ under the equivalence relation of conjugacy of subgroups of $G / N$. Define

$$
S_{G / N}:=\left\{\left(D / N, A_{N} / N\right) \mid D / N \in T_{G / N}, D / N \text { core-free in } G / N\right\} .
$$

Let

$$
S:=\left\{\left(N, D / N, A_{N} / N\right) \mid N \triangleleft G, S_{G / N} \neq \phi,\left(D / N, A_{N} / N\right) \in S_{G / N}\right\} .
$$

We are now ready to recall the theorem describing the complete algebraic structure of semisimple finite metabelian group algebras:

Theorem 1 [3]: Let $G$ be a finite metabelian group of order coprime to $q$. Then,

(i) A complete set of primitive central idempotents of semisimple group algebra $\mathbb{F}_{q}[G]$ is given by the set $\left\{e_{C}\left(G, A_{N}, D\right) \mid\left(N, D / N, A_{N} / N\right) \in S, C \in \mathfrak{R}\left(A_{N} / D\right)\right\}$;

(ii) the simple component corresponding to primitive central idempotent $e_{C}\left(G, A_{N}, D\right)$ is

$$
F_{q}[G] e_{C}\left(G, A_{N}, D\right) \cong M_{\left[G: A_{N}\right]}\left(F_{q^{o\left(A_{N}, D\right)}}\right),
$$

where $M_{n}(R)$ denotes the ring of $n \times n$ matrices over the $\operatorname{ring} R$ and $o\left(A_{N}, D\right)=\frac{\operatorname{ord}_{\left(\left[A_{N}: D\right]\right)}(q)}{\left[E_{G}\left(A_{N}, D\right): A_{N}\right]}$. Moreover the number of such simple components is $\left|\mathfrak{R}\left(A_{N}, D\right)\right|$.

\section{Groups whose central quotient is abelian (not cyclic) group of order $p^{2}$}

Conelissen and Milies [6] have classified indecomposable finitely generated groups $G$, such that $G / Z(G) \cong C_{p} \times C_{p}$, into nine classes. In all of these classes, $G=\langle a, b, Z(G)\rangle$ with some more relations as described in following table: 


\begin{tabular}{|c|c|c|}
\hline Group $G$ & $Z(G)$ & Relations \\
\hline $\mathfrak{G}_{1}$ & $\langle c\rangle$ & $\begin{array}{l}a^{p}, b^{p}, c^{p^{m}}, b^{-1} a^{-1} b a c^{-\left(p^{m-1}\right)}, \\
m \geq 1\end{array}$ \\
\hline $\mathfrak{G}_{2}$ & $\langle c\rangle$ & $\begin{array}{l}a^{p} c^{-1}, b^{p} c^{-1}, c^{p^{m}}, b^{-1} a^{-1} b a c^{-\left(p^{m-1}\right)}, \\
m \geq 1\end{array}$ \\
\hline $\mathfrak{G}_{3}$ & $\left\langle c_{1}\right\rangle \times\left\langle c_{2}\right\rangle$ & $\begin{array}{l}a^{p}, b^{p} c_{2}^{-1}, c_{1}^{p^{m_{1}}}, c_{2}^{p^{m_{2}}}, b^{-1} a^{-1} b a c_{1}^{-\left(p^{m_{-1}-1}\right)} \\
m_{1}, m_{2} \geq 1\end{array}$ \\
\hline $\mathfrak{G}_{4}$ & $\left\langle c_{1}\right\rangle \times\left\langle c_{2}\right\rangle$ & $\begin{array}{l}a^{p} c_{1}^{-1}, b^{p} c_{2}^{-1}, c_{1}^{p^{m_{1}}}, c_{2}^{p^{m_{2}}}, b^{-1} a^{-1} b a c_{1}^{-\left(p^{m_{1}-1}\right)}, \\
m_{1}, m_{2} \geq 1\end{array}$ \\
\hline $\mathfrak{G}_{5}$ & $\left\langle c_{1}\right\rangle \times\left\langle u_{1}\right\rangle$ & $\begin{array}{l}a^{p}, b^{p} u_{1}^{-1}, c_{1}^{p^{m_{1}}}, b^{-1} a^{-1} b a c_{1}^{-\left(p^{m-1}\right)} \\
m_{1} \geq 1\end{array}$ \\
\hline $\mathfrak{G}_{6}$ & $\left\langle c_{1}\right\rangle \times\left\langle u_{1}\right\rangle$ & $\begin{array}{l}a^{p} c_{1}^{-1}, b^{p} u_{1}^{-1}, c_{1}^{p^{m_{1}}}, b^{-1} a^{-1} b a c_{1}^{-\left(p^{m_{1}-1}\right)} \\
m_{1} \geq 1\end{array}$ \\
\hline $\mathfrak{G}_{7}$ & $\left\langle c_{1}\right\rangle \times\left\langle c_{2}\right\rangle \times\left\langle c_{3}\right\rangle$ & $\begin{array}{l}a^{p} c_{2}^{-1}, b^{p} c_{3}^{-1}, c_{1}^{p^{m_{1}}}, c_{2}^{p^{m_{2}}}, c_{3}^{p^{m_{3}}}, b^{-1} a^{-1} b a c_{1}^{-\left(p^{m_{1}-1}\right)}, \\
m_{1}, m_{2}, m_{3} \geq 1\end{array}$ \\
\hline $\mathfrak{G}_{8}$ & $\left\langle c_{1}\right\rangle \times\left\langle c_{2}\right\rangle \times\left\langle u_{1}\right\rangle$ & $\begin{array}{l}a^{p} c_{2}^{-1}, b^{p} u_{1}^{-1}, c_{1}^{p^{m_{1}}}, c_{2}^{p^{m_{2}}}, b^{-1} a^{-1} b a c_{1}^{-\left(p^{m_{-1}-1}\right)}, \\
m_{1}, m_{2} \geq 1\end{array}$ \\
\hline $\mathfrak{G}_{9}$ & $\left\langle c_{1}\right\rangle \times\left\langle u_{1}\right\rangle \times\left\langle u_{2}\right\rangle$ & $\begin{array}{l}a^{p} u_{1}^{-1}, b^{p} u_{2}^{-1}, c_{1}^{p^{m_{1}}}, b^{-1} a^{-1} b a c_{1}^{-\left(p^{m_{1}-1}\right)} \\
m_{1} \geq 1\end{array}$ \\
\hline
\end{tabular}

$o\left(u_{i}\right), i=1,2$ is infinite.

It can be see easily that $G$ is finite metabelian group only in five classes. Out of these five classes, we will give a complete algebraic structure off $\mathbb{F}_{q}[G]$, for $G=\mathfrak{G}_{1}$ and $\mathfrak{G}_{2}$ only. The rest of the cases can be dealt similarly. Throughout this section $\mathbb{F}_{q}$ is a finite field with $q$ elements and $\operatorname{gcd}(p, q)=1$. Let $\operatorname{ord}_{p}(q)$, the order of $q$ modulo $p$, be $f$ and $e=\frac{p-1}{f}$. Write $q^{f}=1+p^{d} c$, where $p$ does not divide $c$. Then for $l \geq 1$,

$$
\operatorname{ord}_{p^{1}}(q)= \begin{cases}f, & l \leq d, \\ f p^{l-d}, & l \geq d+1 .\end{cases}
$$

\subsection{Structure of $\mathbb{F}_{q}\left[\mathfrak{G}_{1}\right]$}

Let $G$ be a group of type $\mathfrak{G}_{1}$. Thus $G$ has following representation:

$$
\left.G=\langle a, b, c| a^{p}=b^{p}=c^{p^{m}}=1, b^{-1} a^{-1} b a=c^{p^{m-1}}, c \text { central in } G\right\rangle(1)
$$


where $p$ is prime and $m \geq 1$. For $p=2$, the complete algebraic structure of $\mathbb{F}_{q}[G]$ can be read from [3]. Suppose $p$ is an odd prime. For $m \geq 2$, define

$$
\begin{gathered}
K_{0}:=\langle 1\rangle, K_{1}:=\langle c, a\rangle, K_{2}^{(i)}:=\left\langle c, a^{i} b\right\rangle, K_{3}^{(i)}:=\left\langle a, b, c^{p^{i}}\right\rangle, 0 \leq i \leq p-1, \\
K_{4}^{(i, j)}:=\left\langle a, c^{p^{i}} b^{j}\right\rangle, K_{5}^{(i, j)}:=\left\langle b, c^{p^{i}} a^{j}\right\rangle, K_{6}^{(i, j, k)}:=\left\langle c^{p^{i}} a^{j}, c^{p^{i}} b^{k}\right\rangle, \\
0 \leq i \leq m-2,1 \leq j, k \leq p-1 .
\end{gathered}
$$

Theorem 2. Acomplete set of primitive central idempotents of semisimple group algebra $\mathbb{F}_{q}[G]$, $G$ of type $\mathfrak{G}_{1}$, is given as follows:

\section{Primitive central idempotents of $\mathbb{F}_{q}[G]$ for $m=1$ :}

$e_{C}(G, G, G), C \in \mathfrak{R}(G / G)$;

$e_{C}\left(G, G,\left\langle c, a^{i} b\right\rangle\right), C \in \mathfrak{R}\left(G /\left\langle c, a^{i} b\right\rangle\right), 0 \leq i \leq p-1 ;$

$e_{C}(G, G,\langle a, c\rangle), C \in \mathfrak{R}(G /\langle a, c\rangle)$;

$e_{C}(G,\langle a, c\rangle,\langle a\rangle), C \in \mathfrak{R}(\langle a, c\rangle /\langle a\rangle)$.

Primitive central idempotents of $\mathbb{F}_{q}[G]$ for $m \geq 2$ :

$e_{C}\left(G, K_{1},\langle a\rangle\right), C \in \mathfrak{R}\left(K_{1} /\langle a\rangle\right) ;$

$e_{C}\left(G, G, K_{1}\right), C \in \mathfrak{R}\left(G / K_{1}\right)$;

$e_{C}\left(G, G, K_{2}^{(i)}\right), C \in \mathfrak{R}\left(G / K_{2}^{(i)}\right) 0 \leq i \leq p-1$;

$e_{C}\left(G, G, K_{3}{ }^{(i)}\right), C \in \mathfrak{R}\left(G / K_{3}{ }^{(i)}\right) \quad 0 \leq i \leq p-1$;

$e_{C}\left(G, G, K_{4}^{(i, j)}\right), C \in \mathfrak{R}\left(G / K_{4}^{(i, j)}\right) \quad 0 \leq i \leq m-2,1 \leq j \leq p-1$;

$e_{C}\left(G, G, K_{5}^{(i, j)}\right), C \in \mathfrak{R}\left(G / K_{5}^{(i, j)}\right) \quad 0 \leq i \leq m-2,1 \leq j \leq p-1$;

$e_{C}\left(G, G, K_{6}^{(i, j, k)}\right), C \in \mathfrak{R}\left(G / K_{6}{ }^{(i, j, k)}\right) \quad 0 \leq i \leq m-2,1 \leq j, k \leq p-1$.

To prove this Theorem, we first need to find the normal subgroups of $G$.

Lemma 1. Let $G$ be a group as defined in (1) and $\mathcal{N}$ be the set of distinct normal subgroups of $G$. Then

(i) For $\mathrm{m}=1, \mathcal{N}=\left\{\langle 1\rangle,\langle c\rangle,\langle c, a\rangle,\langle c, a, b\rangle\left\langle c, a^{i} b\right\rangle, 0 \leq i \leq p-1\right\}$

and $S=\{(\langle 1\rangle,\langle a\rangle,\langle a, c\rangle)\} \cup\{(\langle c, a\rangle,\langle 1\rangle, G /\langle c, a\rangle)\} \cup\{(\langle G\rangle,\langle 1\rangle,\langle 1\rangle)\} \cup$

$\left\{\left(\left\langle c, a^{i} b\right\rangle,\langle 1\rangle, G /\left\langle c, a^{i} b\right\rangle \mid 0 \leq i \leq p-1\right)\right\} ;$

(ii) For $m \geq 2, \mathcal{N}=\left\{\left\langle c^{p^{i}}\right\rangle,\left\langle c^{p^{i}}, a\right\rangle,\left\langle c^{p^{i}}, b\right\rangle,\left\langle c^{p^{i}}, a, b\right\rangle \mid 0 \leq i \leq m-1\right\} \cup$

$$
\begin{aligned}
& \left\{\left\langle c^{p^{m-1}}, a^{j} b\right\rangle,\left\langle c^{p^{i}} a^{j}\right\rangle,\left\langle c^{p^{i}} b^{j}\right\rangle,\left\langle c^{p^{i}}, a^{j} b\right\rangle,\left\langle a, c^{p^{i}} b^{j}\right\rangle,\left\langle b, c^{p^{i}} a^{j}\right\rangle \mid 0 \leq i \leq m-2,\right. \\
& 1 \leq j \leq p-1\} \cup\left\{\left\langle c^{p^{i}} a^{k j} b^{j}\right\rangle,\left\langle c^{p^{i}} a^{j}, c^{p^{i}} b^{k}\right\rangle \mid 0 \leq i \leq m-2,1 \leq j, k \leq p-1\right\} \\
& \text { and } S=\left\{\left(K_{0},\langle a\rangle, K_{1}\right)\right\} \cup\left\{\left(K_{1}, K_{0}, G / K_{1}\right)\right\} \cup\left\{\left(K_{2}^{(i)}, K_{0}, G / K_{2}^{(i)}\right) \mid 0 \leq i \leq p-1\right\} \cup \\
& \left\{\left(K_{3}^{(i)}, K_{0}, G / K_{3}^{(i)}\right) \mid 0 \leq i \leq p-1\right\} \cup\left\{\left(K_{4}^{(i, j)}, K_{0}, G / K_{4}^{(i, j)}\right) \mid 0 \leq i \leq m-2,1 \leq j\right. \\
& \leq p-1\} \cup\left\{\left(K_{5}^{(i, j)}, K_{0}, G / K_{5}^{(i, j)}\right) \mid 0 \leq i \leq m-2,1 \leq j \leq p-1\right\} \cup\left\{\left(K_{6}^{(i, j, k)}, K_{0}, G / K_{6}^{(i, j, k)}\right) \mid\right. \\
0 \leq i & \leq m-2,1 \leq j, k \leq p-1\} .
\end{aligned}
$$


Proof. It can be seen easily that in (i) and (ii), the subgroups listed are distinct and normal in $G$. Also if $N \triangleleft G$, then it can be shown easily, as in [[3], Lemma 4], that $N$ is one of the subgroups listed in the statement of Lemma.

Observe that in both (i) and (ii), for $N=\langle 1\rangle, A_{N} / N=\langle c, a\rangle$. Hence $S_{G / N}=\{(\langle a\rangle,\langle c, a\rangle)\}$. Moreover for non-identity normal subgroup $N$ of $G$, the derived group of $G, G^{\prime}=\left\langle c^{p^{m-1}}\right\rangle$ is contained in $N$, thus $G / N$ is abelian and hence $A_{N} / N=G / N$. Thus for all non identity normal subgroups $N$ of $G$,

$$
S_{G / N}= \begin{cases}\{(\langle 1\rangle, G / N)\}, & \text { if } G / N \text { is cyclic } \\ \phi, & \text { otherwise. }\end{cases}
$$

Thus to complete the proof, we need to find only those $N \in \mathcal{N}$ for which $G / N$, is cyclic. In (i), the subgroups $\langle c, a\rangle,\langle c, a, b\rangle,\left\langle c, a^{i} b\right\rangle, 0 \leq i \leq p-1$ have cyclic quotient with $G$, whereas in (ii), the following normal subgroups have cyclic quotient with $G$ :

$$
\begin{gathered}
K_{1}, K_{2}^{(i)}, K_{3}^{(i)}, 0 \leq i \leq p-1, \\
K_{4}^{(i, j)}, K_{5}^{(i, j)}, K_{6}^{(i, j, k)}, 0 \leq i \leq m-2,1 \leq j, k \leq p-1 .
\end{gathered}
$$

Thus the proof of the lemma is complete.

Proof of Theorem 2. The list of primitive central idempotents of group algebra $\mathbb{F}_{q}[G]$ can now be easily obtained with the help of Theorem 1 and Lemma 1.

Theorem 3. TheWedderburn decomposition and the automorphism group of semisimple group algebra $\mathbb{F}_{q}[G], G$ of type $\mathfrak{b}_{1}$, are given as follows:

\section{Wedderburn decomposition}

$$
\mathbb{F}_{q}[G] \cong\left\{\begin{array}{cc}
\mathbb{F}_{q} \oplus \mathbb{F}_{q^{f}}((p+1) e) \oplus M_{p}\left(\mathbb{F}_{q^{f}}\right)^{(e)}, & m=1, \\
\mathbb{F}_{q} \oplus \mathbb{F}_{q^{f}}\left(\frac{p^{m+1}-1}{f}\right) \oplus M_{p}\left(\mathbb{F}_{q^{f}}\right)^{\left(p^{m-1} e\right)}, & 2 \leq m \leq d, \\
\mathbb{F}_{q} \oplus \mathbb{F}_{q^{f}}\left(\frac{p^{d+2}-1}{f}\right)_{\oplus} \sum_{i=d+1}^{m-1} \mathbb{F}_{q^{f p^{i-d}}}\left(p^{d+1} e\right) \oplus M_{p}\left(\mathbb{F}_{q^{f p^{m-d}}}\right)^{\left(p^{d-1} e\right)}, & m \geq d+1 .
\end{array}\right.
$$

Automorphism group

$$
\operatorname{Aut}\left(\mathbb{F}_{q}[G]\right) \cong\left\{\begin{array}{c}
\left(\mathbb{Z}_{f}^{((p+1) e)} \ltimes S_{(p+1) e}\right) \oplus\left(\left(S L_{p}\left(\mathbb{F}_{q^{f}}\right) \ltimes \mathbb{Z}_{f}\right)^{(e)} \ltimes S_{e}\right), m=1, \\
\left(\mathbb{Z}_{f}\left(\frac{p^{m+1}-1}{f}\right) \ltimes S_{\left(\frac{p^{m+1}-1}{f}\right)}\right) \oplus \\
\left(\left(S L_{p}\left(\mathbb{F}_{q}\right) \ltimes \mathbb{Z}_{f}\right)^{\left(p^{m-1} e\right)} \ltimes S_{p^{m-1} e}\right), 2 \leq m \leq d, \\
\left(\mathbb{Z}_{f}^{\left(\frac{p^{d+2}-1}{f}\right)} \ltimes S_{\left(\frac{p^{d+2}-1}{f}\right)} \oplus \sum_{i=d+1}^{m-1}\left(\mathbb{Z}_{f p^{i-d}}\left(p^{d+1}\right) e\right) \ltimes S_{p^{d+1} e}\right) \oplus \\
\left(\left(S L_{p}\left(\mathbb{F}_{q^{f p^{m-d}}}\right) \ltimes \mathbb{Z}_{f p^{m-d}}\right)^{\left(p^{d-1} e\right)} \ltimes S_{p^{d-1} e}\right), m \geq d+1 .
\end{array}\right.
$$

where $\mathbb{Z}_{n}$ denotes the cyclic group of order $n, S_{n}$ denotes the symmetric group of degree $n$ and for a group $H, H^{(n)}$ a direct sum of $n$ copies of $H$. 
Proof of Theorem 3. In order to find the Wedderburn decomposition of $\mathbb{F}_{q}[G]$, we need to find the simple component corresponding to each primitive central idempotent. More precisely, for each $\left(N, D / N, A_{N} / N\right) \in S, C \in \mathfrak{R}\left(A_{N} / D\right)$, we need to calculate $o\left(A_{N}, D\right)$ and $\left|\mathfrak{R}\left(A_{N} / D\right)\right|$, as given by the following tables:

Case I : $m=1$

\begin{tabular}{|c|c|c|c|}
\hline$\left(N, D / N, A_{N} / N\right)$ & $E_{G}\left(A_{N} / D\right)$ & $o\left(A_{N}, D\right)$ & $\left|\mathfrak{R}\left(A_{N} / D\right)\right|$ \\
\hline$(\langle 1\rangle,\langle a\rangle,\langle a, c\rangle)$ & $\langle a, c\rangle$ & $f$ & $e$ \\
\hline$(\langle c, a\rangle,\langle 1\rangle, G /\langle c, a\rangle)$ & $G$ & $f$ & $e$ \\
\hline$(\langle G\rangle,\langle 1\rangle,\langle 1\rangle)$ & $G$ & 1 & 1 \\
\hline $\begin{array}{l}\left(\left\langle c, a^{i} b\right\rangle,\langle 1\rangle, G /\left\langle c, a^{i} b\right\rangle\right) \\
0 \leq i \leq p-1\end{array}$ & $G$ & $f$ & $e$ \\
\hline
\end{tabular}

Case II $: m \geq 2$

\begin{tabular}{|l|l|l|l|}
\hline$\left(N, D / N, A_{N} / N\right)$ & $E_{G}\left(A_{N} / D\right)$ & $o\left(A_{N}, D\right)$ & $\left|\mathfrak{R}\left(A_{N} / D\right)\right|$ \\
\hline$\left(K_{0},\langle a\rangle, K_{1}\right)$ & $K_{1}$ & $\begin{cases}f, & m \leq d, \\
f p^{m-d}, & m \geq d+1 .\end{cases}$ & $\begin{cases}p^{m-1} e, \quad m \leq d, \\
p^{d-1} e, & m \geq d+1 .\end{cases}$ \\
\hline $\begin{array}{l}\left(K_{1}, K_{0}, G / K_{1}\right) \\
\left(K_{2}^{(i)}, K_{0}, G / K_{2}^{(i)}\right)\end{array}$ & $G$ & $f$ & $e$ \\
\hline $\begin{array}{l}\left(K_{3}^{(i)}, K_{0}, G / K_{3}^{(i)}\right) \\
0 \leq i \leq p-1\end{array}$ & $G$ & $\begin{cases}1, & i=0 \\
f, & 1 \leq i \leq d \\
f p^{i-d}, & i \geq d+1\end{cases}$ & $\begin{cases}f, & i \leq d-1, \\
p^{i-1} e, & 1 \leq i \leq d, \\
p^{d-1} e, & i \geq d+1 .\end{cases}$ \\
\hline $\begin{array}{l}\left(K_{4}^{(i, j)}, K_{0}, G / K_{4}^{(i, j)}\right) \\
0 \leq i \leq m-2,\end{array}$ & $G$ & $\begin{cases}p^{i} e, & i \leq d-1, \\
p^{d-1} e, & i \geq d .\end{cases}$ \\
$1 \leq j \leq p-1$ & $\begin{cases}f, & i \leq d-1, \\
f p^{i-d+1}, & i \geq d .\end{cases}$ & $\begin{cases}p^{i} e, & i \leq d-1, \\
p^{d-1} e, & i \geq d .\end{cases}$ \\
\hline $\begin{array}{l}\left(K_{5}^{(i, j)}, K_{0}, G / K_{5}^{(i, j)}\right) \\
0 \leq i \leq m-2, \\
1 \leq j \leq p-1\end{array}$ & $G$ & $\begin{cases}f, & i \leq d-1, \\
f p^{i-d+1}, & i \geq d .\end{cases}$ \\
\hline $\begin{array}{l}\left(K_{6}^{(i, j, k)}, K_{0}, G / K_{6}^{(i, j, k)}\right) \\
0 \leq i \leq m-2, \\
1 \leq j, k \leq p-1\end{array}$ & $G$ & $\begin{cases}p^{i} e, & i \leq d-1, \\
p^{d-1} e, & i \geq d .\end{cases}$ \\
\hline
\end{tabular}

Now, the required Wedderburn decomposition and automorphism group can be easily read from these two tables and [3, Theorem 3]. 


\subsection{Structure of $\mathbb{F}_{q}\left[\mathfrak{G}_{2}\right]$}

Observe that if group $G$ is of type $\mathfrak{G}_{2}$, then it has the following presentation:

$$
\left.G=\langle a, b| a^{p^{m+1}}=1, b^{p}=a^{p}, b^{-1} a^{-1} b a=a^{p^{m}+1}, a^{p} \text { central in } G\right\rangle,
$$

where $p$ is a prime and $m \geq 1$. For $p=2$, the complete algebraic structure of $\mathbb{F}_{q}[G]$ can be read from [3]. Suppose $p$ is an odd prime. For $m \geq 1$, set:

$$
\begin{gathered}
L_{0}:=\langle 1\rangle, L_{1}:=\langle a\rangle, L_{2}{ }^{(i)}:=\left\langle a^{p^{i}}\right\rangle, 1 \leq i \leq m, L_{3}:=\langle a, b\rangle, \\
L_{4}{ }^{(i)}:=\left\langle a^{p}, a^{i} b\right\rangle, 0 \leq i \leq p-1, \\
L_{5}{ }^{(i, j)}:=\left\langle a^{p^{i}}, a^{j p^{i-1}-1} b\right\rangle, 2 \leq i \leq m, 1 \leq j \leq p .
\end{gathered}
$$

The following Theorems give a complete algebraic structure of semisimple group algebra $\mathbb{F}_{q}[G]:$

Theorem 4. A complete set of primitive central idempotents of semisimple group algebra $\mathbb{F}_{q}[G]$, $G$ of type $\mathfrak{G}_{2}$, is given as follows:

$$
\begin{gathered}
\text { Primitive central idempotents of } \mathbb{F}_{q}[G] \\
e_{C}(G, G, G), C \in \mathfrak{R}(G / G) ; \\
e_{C}\left(G, G, L_{1}\right), C \in \mathfrak{R}\left(G / L_{1}\right) ; \\
e_{C}\left(G, G, L_{3}\right), C \in \mathfrak{R}\left(G / L_{3}\right) ; \\
e_{C}\left(G, L_{1}, L_{0}\right), C \in \Re\left(L_{1} / L_{0}\right) ; \\
e_{C}\left(G, G, L_{4}{ }^{(i)}\right), C \in \mathfrak{R}\left(G / L_{4}{ }^{(i)}\right), 0 \leq i \leq p-1 ; \\
e_{C}\left(G, G, L_{5}{ }^{(i, j)}\right), C \in \mathfrak{R}\left(G / L_{5}{ }^{(i, j)}\right), 2 \leq i \leq m, 1 \leq j \leq p .
\end{gathered}
$$

Proof of Theorem 4. In view of Theorem 1, to find a complete list of primitive central idempotents of $\mathbb{F}_{q}[G]$ we first need to list all normal subgroups of $G$. It can be see easily that the set $\mathcal{N}$ of distinct normal subgroups of $G$ is equal to

$$
\left\{L_{0}, L_{1}, L_{2}{ }^{(i)}, 1 \leq i \leq m, L_{3}, L_{4}^{(i)}, 0 \leq i \leq p-1, L_{5}^{(i, j)}, 2 \leq i \leq m, 1 \leq j \leq p\right\} .
$$

For $N=L_{0}, A_{N} / N=L_{1}$. Hence $S_{G / N}=\left\{\left(L_{0}, L_{1}\right)\right\}$. Moreover, for non-identity $N \in \mathcal{N}, S_{G / N}$ is non-empty if and only if $G / N$ is cyclic. The following $N \in \mathcal{N}$ have cyclic quotient with $G$ :

$$
L_{1}, L_{3}, L_{4}^{(i)}, 0 \leq i \leq p-1, L_{5}^{(i, j)}, 2 \leq i \leq m, 1 \leq j \leq p .
$$

Thus (i) follows from Theorem 1.

Theorem 5. The Wedderburn decomposition and the automorphism group of semisimple group algebra $\mathbb{F}_{q}[G], G$ of type $\mathfrak{G}_{2}$, are as follows:

\section{Wedderburn decomposition}

$$
\mathbb{F}_{q}[G] \cong \begin{cases}\mathbb{F}_{q} \oplus \mathbb{F}_{q^{f}}\left(\frac{p^{m+1}-1}{f}\right) \oplus M_{p}\left(\mathbb{F}_{q^{f}}\right)^{\left(p^{m-1} e\right)}, & m \leq d-1, \\ \mathbb{F}_{q} \oplus \mathbb{F}_{q^{f}}\left(\frac{p^{d+1}-1}{f}\right) \oplus \sum_{i=d+1}^{m} \mathbb{F}_{q^{f p^{i-d}}}\left(p^{d} e\right) \oplus M_{p}\left(\mathbb{F}_{q^{f p^{m-d}}}\right)^{\left(p^{d-1} e\right)}, \quad m \geq d .\end{cases}
$$




$$
\begin{gathered}
\text { Automorphism group } \\
\operatorname{Aut}\left(\mathbb{F}_{q}[G]\right) \cong\left\{\begin{array}{c}
\left(\mathbb{Z}_{f}\left(\frac{p^{m+1}-1}{f}\right) \ltimes S_{\left(\frac{p^{m+1}-1}{f}\right)}\right) \oplus \\
\left.\left(S L_{p}\left(\mathbb{F}_{q^{f}}\right) \ltimes \mathbb{Z}_{f}\right)^{\left(p^{m-1} e\right)} \ltimes S_{p^{m-1} e}\right), m \leq d-1 \\
\left.\left(\mathbb{Z}_{f}\left(\frac{p^{d+1}-1}{f}\right) \ltimes S_{\left(\frac{p^{d+1}-1}{f}\right)}\right) \oplus \sum_{i=d+1}^{m}\left(\mathbb{Z}_{f p^{i-d}}\left(p^{d}\right) e\right) \ltimes S_{p^{d}}\right) \oplus \\
\left(\left(S L_{p}\left(\mathbb{F}_{q^{f p^{m-d}}}\right) \ltimes \mathbb{Z}_{f p^{m-d}}\right)^{\left(p^{d-1} e\right)} \ltimes S_{p^{d-1} e}\right), m \geq d .
\end{array}\right.
\end{gathered}
$$

Proof of Theorem 5. We will first find $E_{G}\left(L_{1} / L_{0}\right)$.Observe that $\left|L_{1} / L_{0}\right|=p^{m+1}$ and $L_{1} \subseteq E_{G}\left(L_{1} / L_{0}\right) \subseteq G$. Let $m \leq d-1$. In this case, $b \in E_{G}\left(L_{1} / L_{0}\right)$, if and only if $\zeta^{p^{m+1}}=\zeta^{q^{i}}$ for some $i, 1 \leq i \leq f$, where $\zeta$ is a primitive $p^{m+1}$ th root of unity. This implies that $p^{m}+1=q^{i}\left(\bmod p^{m+1}\right)$, i.e., $p=\frac{f}{\operatorname{gcd}(i, f)}$, which gives that $p$ divides $p-1$, a contradiction.

\begin{tabular}{|c|c|c|c|}
\hline$\left(N, D / N, A_{N} / N\right)$ & $E_{G}\left(A_{N} / D\right)$ & $o\left(A_{N}, D\right)$ & $\left|\mathfrak{R}\left(A_{N} / D\right)\right|$ \\
\hline$\left(G, L_{0}, L_{0}\right)$ & $G$ & 1 & 1 \\
\hline$\left(L_{1}, L_{0}, G / L_{1}\right)$ & $G$ & $f$ & $e$ \\
\hline $\begin{array}{l}\left(L_{4}^{(i)}, L_{0}, G / L_{4}^{(i)}\right) \\
0 \leq i \leq p-1\end{array}$ & $G$ & $f$ & $e$ \\
\hline $\begin{array}{l}\left(L_{5}^{(i, j)}, L_{0}, G / L_{5}^{(i, j)}\right), \\
2 \leq i \leq m, 1 \leq j \leq p\end{array}$ & $G$ & $\begin{cases}f, & i \leq d \\
f p^{i-d}, & i \geq d+1\end{cases}$ & $\left\{\begin{array}{l}p^{i-1} e, \quad i \leq d \\
p^{d-1} e, \quad i \geq d+1\end{array}\right.$ \\
\hline$\left(L_{0}, L_{0}, L_{1}\right)$ & $\begin{cases}L_{1}, & m \leq d-1 \\
G, & m \geq d\end{cases}$ & $\begin{cases}f, & m \leq d-1, \\
f p^{m-d}, & m \geq d\end{cases}$ & $\begin{cases}p^{m-1} e, & m \leq d-1, \\
p^{d-1} e, & m \geq d .\end{cases}$ \\
\hline
\end{tabular}
Hence in this case $E_{G}\left(L_{1} / L_{0}\right)=L_{1}$. For $m \geq d, E_{G}\left(L_{1} / L_{0}\right)=G$. Thus we have the following:

The Wedderburn decomposition and automorphism group of $\mathbb{F}_{q}[G]$ can now be easily read with the help of this table and [3, Theorem 3].

\section{Acknowledgment}

The author is grateful to the referees for their valuable suggestions which have helped to write the paper in the present form. 


\section{References}

[1] G.K. Bakshi, S. Gupta, I.B.S. Passi, Semisimple metacyclic group algebras, Proc. Indian Acad.Sci.(Math Sci.). 121(4) (2011) 379-396.

[2] G.K. Bakshi, S. Gupta, I.B.S. Passi, The structure of finite Semisimple metacyclic group algebras, J. Ramanujan Math Soc. 28(2) (2013) 141-158.

[3] G.K. Bakshi, S. Gupta, I.B.S. Passi, The algebraic structure of Finite metabelian group algebras, Comm. Algebra. 43(6) (2015) 2240-2257.

[4] G.K. Bakshi, R.S. Kullkarni, I.B.S. Passi, The rational group algebra of a finite group, J. Alg. Appl. 12(3) (2013).

[5] O. Broche, Á. del Río, Wedderburn decomposition of finite group Algebras, Finite Fields Appl. 13(1) (2007) 71-79.

[6] M.G. Cornelissen, P.C. Milies, Finitely generated groups $\mathrm{G}$ such that $G / \mathrm{Z}(G) \cong C_{p} \times C_{p}$, Comm. Algebra. 42(1) (2014) 378-388.

[7] E. Jespers, G. Leal, A. Paques, Central idempotents in the rational group algebra of a finite nilpotent group, J. Algebra Appl. 2(1) (2003) 57-62.

[8] A. Olivieri, Á. del Río, J.J. Simón, The group of automorphisms of the rational group algebra of a finite metacyclic group, Comm. Algebra. 34(10) (2006) 3543-3567.

[9] A. Olivieri, Á. del Río, J.J. Simón, On monomial characters and Central idempotents of rational group algebras, Comm. Algebra. 32(4) (2004) 1531-1550. 